\title{
Tissue Factor in Dermatitis Herpetiformis and Bullous Pemphigoid: Link between Immune and Coagulation System in Subepidermal Autoimmune Bullous Diseases
}

\author{
Agnieszka Zebrowska, ${ }^{1}$ Malgorzata Wagrowska-Danilewicz, ${ }^{2}$ \\ Marian Danilewicz, ${ }^{2}$ Joanna Wieczfinska, ${ }^{3}$ Ewa Pniewska, ${ }^{3}$ Michal Zebrowski, ${ }^{4}$ \\ Elzbieta Waszczykowska, ${ }^{1}$ Anna Wozniacka, ${ }^{1}$ Makandjou-Ola Eusebio, ${ }^{5}$ \\ Miroslawa Pietruczuk, ${ }^{5}$ and Rafal Pawliczak ${ }^{3}$ \\ ${ }^{1}$ Department of Dermatology and Venereology, Medical University of Lodz, Hallera Square 1, 90-497 Lodz, Poland \\ ${ }^{2}$ Laboratory of Nephropathology, Medical University of Lodz, Pomorska 251 Street, 92-213 Lodz, Poland \\ ${ }^{3}$ Department of Immunopathology, Faculty of Biomedical Sciences and Postgraduate Training, Medical University of Lodz, \\ Zeligowskiego 7/9, 90-752 Lodz, Poland \\ ${ }^{4}$ Department of Social Medicine, Medical University of Lodz, Zeligowskiego 7/9, 90-752 Lodz, Poland \\ ${ }^{5}$ Department of Laboratory Diagnostics, Medical University of Lodz, Kopcinskiego 22, 90-153 Lodz, Poland
}

Correspondence should be addressed to Agnieszka Zebrowska; zebrowskaaga@wp.pl

Received 30 August 2015; Revised 27 November 2015; Accepted 10 December 2015

Academic Editor: Sandra Helena Penha Oliveira

Copyright ( 2015 Agnieszka Zebrowska et al. This is an open access article distributed under the Creative Commons Attribution License, which permits unrestricted use, distribution, and reproduction in any medium, provided the original work is properly cited.

\begin{abstract}
Dermatitis herpetiformis (DH) and bullous pemphigoid (BP) are skin diseases associated with eosinophilic and neutrophilic infiltrations. Although chemokines are critical for the selective accumulation and activation of various leukocyte subsets in the inflammatory process, there are few findings concerning inflammatory cells and production of coagulation factors in blistering diseases. Skin biopsies were taken from 14 patients with DH, 27 with BP, and 20 control subjects. The localization and expression of tissue factor (TF) in skin lesions and perilesional skin were studied by immunohistochemistry and confirmed by Western Blot. Moreover the plasma concentrations of TF were measured by immunoassays. D dimers, fibrinogen, and selected coagulation parameters were measured by routine methods. Expression of TF in the epidermis and in inflammatory influxed cells in dermis was detected in skin biopsies from BP patients. Examined TF expression was detected in perilesional skin of all BP patients too. The expression of TF was not observed in biopsies from healthy people and DH patients. The findings of the study show an increased expression of tissue factor in the lesional and perilesional skin of patients with bullous pemphigoid. The difference in chemokine pattern expression and variations in the cellular infiltration in $\mathrm{BP}$ and $\mathrm{DH}$ cause variable expression of TF.
\end{abstract}

\section{Introduction}

Dermatitis herpetiformis (DH) is one of the subepidermal autoimmune bullous diseases (ABD) characterized by skin and intestinal lesions. Skin lesions include polymorphic eruption accompanied by severe pruritus [1]. Diagnosis of $\mathrm{DH}$ is established based on the results of direct immunofluorescence test (DIF) and the presence of circulating IgA antibodies directed against endomysium and/or tissue and epidermal transglutaminase (tTG, eTG) $[2,3]$. Skin lesions in
DH are histologically characterized by neutrophilic infiltrate leading to destruction of basement membrane zone (BMZ) proteins, anchoring fibers, and blister formation [4-6].

Bullous pemphigoid (BP) is a blistering disease, characterized by inflammatory infiltrate in the dermis, presence of IgG and C3 deposits along the basement membrane zone, and circulating IgG autoantibodies. Autoantibodies binding to autoantigens (BPAG1 and BPAG2) localized in the basement membrane of the epidermis activate a series of immunological and enzymatic phenomena leading to destruction of BMZ 
components and anchoring fibers and blister formation as in $\mathrm{DH}[7,8]$.

Ultrastructural studies confirmed also the presence of intensive inflammatory infiltrate at dermoepidermal junction, as well as destruction of components of extracellular matrix in BP and DH [9-11].

In the available literature, there are few reports on the expression of procoagulant factors, abnormal activation of coagulation process, and their role in the creation of blisters. Recently, the dominant role of eosinophils in the inflammatory infiltrate in bullous diseases is postulated. Therefore, the fact that they might be an important source of procoagulant tissue factor (TF) gives the scientific basis to consider its contribution of this process in the pathogenesis of lesions in the subepidermal bullous diseases.

Recent studies have shown an increased risk of fatal thrombotic events in patients with BP treated with glucocorticoids [12]. Confirmation of these complications is the increased concentration of $\mathrm{D}$ dimers and prothrombin in the serum of patients in the active stage of the disease [13]. Therefore, it is important to define the role of procoagulant factors in the destruction of the basement membrane in pemphigoid and activity of eosinophils and mediators of these cells in skin lesions and normal looking skin as well as in serum in the active phase of the disease.

Under physiological conditions, there is a balance between the coagulation and fibrinolysis, but in pathological states this balance might be deteriorated. In bullous diseases inflammation disrupts homeostatic system and moves it towards the prothrombotic status. Rico et al. [14] describe the dominance of the Th2 cytokine profile in blistering diseases. Interleukin-5, produced mainly by Th2 lymphocytes, is a key factor in the differentiation and activation of eosinophils [14$16]$.

Inflammatory cells are important factors in the coagulation system. Eosinophils, the dominant inflammatory cells in $\mathrm{BP}$, seem to be a major source of intravascular tissue factor [17]. TF is the primary activator of extrinsic coagulation pathway $[17,18]$.

Coagulation pathway might cause the activation of adhesion molecules expression and subsequent release inflammatory mediators and proteolytic enzymes [10, 19]. Recent experimental studies have also shown that these processes may also activate procoagulant factors that cause the development of blood clots, which are the most common cause of complications in patients with BP [20].

TF, in addition to well-documented prothrombotic properties, plays an important role in the inflammatory process. Constitutive expression of TF is present on fibroblasts and epithelial cells, while on monocytes and macrophages it is induced by antigen-antibody complexes and complement activation products and proteins as well as by inflammatory cytokines $[21,22]$.

The goal of this study was to assess the activity of procoagulant factors and their impact on the inflammatory infiltrate in BP and DH before treatment. The data might be an important therapeutic indication allowing for alleviating the high mortality rate among patients with pemphigoid treated with corticosteroids and confirming a new trend in the treatment of BP.

\section{Materials and Methods}

2.1. Patients. The study group involved 41 patients, including 27 patients with BP (15 women and 12 men, age range: 58 to 84 years, mean age: 68.5 years) and 14 patients with $\mathrm{DH}$ (6 women and 8 men, age range: 18 to 70 years, mean age: 49.8 years) in the active phase of the disease. The control group consisted of 20 healthy subjects ( 5 women and 5 men, age range: $50-80$ years, mean age: 71.6 for BP patients and 6 women and 4 men, age range: $19-49$ years, mean age: 42.0 years for $\mathrm{DH}$ patients). In $\mathrm{DH}$ patients direct immunofluorescence tests revealed the presence of granular deposits of IgA in skin papillae and indirect immunofluorescence tests were positive for IgAEmA in all the patients (titer 1:401:640, median 1:80). Anti-tissue transglutaminase antibodies measured using an immunoassay were present in 7/10 cases (median: $5.1 \mathrm{IU} / \mathrm{mL}$ (range: $0.0-186.3 ; \mathrm{IU} / \mathrm{mL}$ )).

The BP patients were at an active stage of the disease and the average score on BPDAI (Bullous Pemphigoid Disease Activity Index) was $38 \pm 11$. The histopathologic findings according to Ackerman in all cases were fully developed. In all the patients direct immunofluorescence test revealed IgG/C3 linear deposits along BMZ. In salt split test deposits were observed in epidermal part of the blister. Using indirect immunofluorescence test circulating IgG antibodies were found in $9 / 14$ patients. ELISA test showed the anti-NC16 autoantibodies present in serum of 11 out of 14 patients.

All the patients signed informed consent before entering the study and the study protocol (\#RNN/93/03/KE) was approved by The Local Ethical Committee of Medical University of Lodz. From all patients in the study group and the healthy control group the blood was collected to several samples. In all patients the blood was collected during active stage of disease without any administered treatment. Skin biopsies from skin lesions were taken for histopathological examination in the study groups and from the healthy skin in the neck area in control group.

2.2. Immunohistochemistry. Paraffin-embedded sections were used for routine $\mathrm{H}+\mathrm{E}$ staining and for immunohistochemistry with DAKO EnVision detection system using immunoperoxidase method. The following primary monoclonal antibodies were used: anti-tissue factor obtained from American Diagnostica Inc., Greenwich, CT, USA, according to manufacturers' instruction.

2.3. Morphometry. Histological morphometry was performed by means of image analysis system consisting of a PC computer equipped with a Pentagram graphical tablet, Indeo Fast card, produced by Indeo (Taiwan), and color TV camera Panasonic (Japan) coupled to a Carl Zeiss microscope (Germany). The TF positive cells were counted in a sequence of $7-10$ consecutive computer images of $400 \mathrm{x}$ high power fields, $0.0047 \mathrm{~mm}^{2}$ each. The results were expressed as percentages 
of TF positive cells of all lymphocytes determined by their morphology.

2.4. Immunoassay. TF levels were measured in plasma in all patients and healthy controls undergoing skin biopsy. Five $\mathrm{mL}$ of venous blood was drawn from the ulnary vein and after centrifugation serum was stored at $-20^{\circ} \mathrm{C}$ for an immunoassay. The enzyme-linked immunoassays were used to measure TF-IMUBIND Tissue Factor ELISA Kit (American Diagnostica Inc.). D dimers levels were performed, using the optical test STA Compact (Roche Diagnostics); fibrinogen levels were measured using chronometric method of Clauss and turbidity method; coagulogram was done according to the standard procedure; DD (D dimers), F2 (factor II), F5 (factor V), F7 (factor VII), and venom (snake venom factor $\mathrm{V}$ activator) were assessed by Destiny Plus in BP patients (Horiba ABX).

2.5. Immunoblotting. Total protein from frozen skin samples was extracted in RIPA protein extraction buffer, supplemented with protease inhibitor cocktail (Sigma-Aldrich, St. Louis, MO, USA). The lysate was centrifuged at $14,000 \times \mathrm{g}$ for $20 \mathrm{~min}$ and the pellet was discarded. Protein concentrations were determined by the BCA Protein Assay Kit (Pierce Thermo Scientific, USA). Electrophoresis was performed in SDS-NuPAGE Gels (Life Technologies, California), subsequently transferred to a nitrocellulose. The membrane was blocked and incubated with the mouse monoclonal anti-TF (Merck Millipore, Billerica, USA). Afterwards the membrane was washed with TBST and incubated in PBST containing the goat anti-mouse IgG secondary antibodies conjugated with alkaline phosphatase (Merck Millipore, USA). The bands were developed using BCIP/NBT Alkaline Phosphatase Substrate (Merck Millipore, Billerica, USA), analyzed with ImageJ 1.34s software (Wayne Rasband, National Institutes of Health, Bethesda), and expressed as optical density (OD).

2.6. Statistical Methods. All values were expressed as mean \pm SEM. Differences between groups were tested using unpaired Student's $t$-test proceeded by the evaluation of normality. The Mann-Whitney $U$ test was used where appropriate. Results were considered statistically significant if $p<0.05$. The data of immunoblotting were analyzed with Student's $t$-test and Mann-Whitney $U$ test. Levene's test was used to assess homogeneity of variances. The level of significance was defined as $p<0.05$.

\section{Results}

3.1. Immunohistochemistry: Tissue Factor. TF expression was demonstrated in cells in the inflammatory infiltrates (eosinophils) and keratinocytes and in the blister fluid (Figure 1). In patients with BP, the expression of TF was also shown in the perilesional skin (in 17 out of 27 individuals) (Figure 2). In patients with BP, in skin lesions, a high expression of TF has been demonstrated mainly in keratinocytes of the basal layer (in 20 out of 27 biopsies) and also in other
TABLE 1: Morphometric analysis of TF expression in skin lesions and in perilesional skin (in keratinocytes and infiltrates) in patients with BP. Immunohistochemistry.

\begin{tabular}{lc}
\hline & Mean \pm SEM \\
\hline BP skin lesions $(n=27)$ & $1.34 \pm 0.62$ \\
BP perilesional skin $(n=27)$ & $0.92 \pm 0.54$ \\
Control group $(n=10)$ & $0.16 \pm 0.28$ \\
DH skin lesions $(n=14)$ & $0.18 \pm 0.24$ \\
DH perilesional skin $(n=14)$ & $0.15 \pm 0.22$ \\
\hline BP skin lesions versus BP perilesional skin & $p<0.02$ \\
BP skin lesions versus control group & $p<0.001$ \\
DH skin lesions versus perilesional skin DH & ND \\
BP skin lesions versus DH skin lesions & $p<0.05$ \\
BP perilesional skin versus DH perilesional skin & $p<0.05$ \\
\hline
\end{tabular}

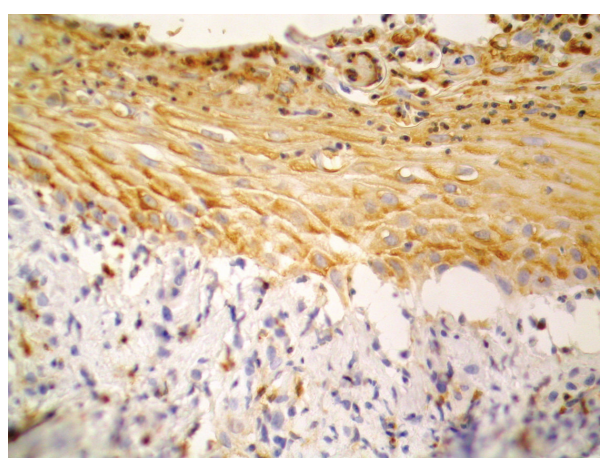

FIGURE 1: Expression of TF in epidermis and cells of influx. Skin lesions. Bullous pemphigoid. 400x Immunohistochemistry.

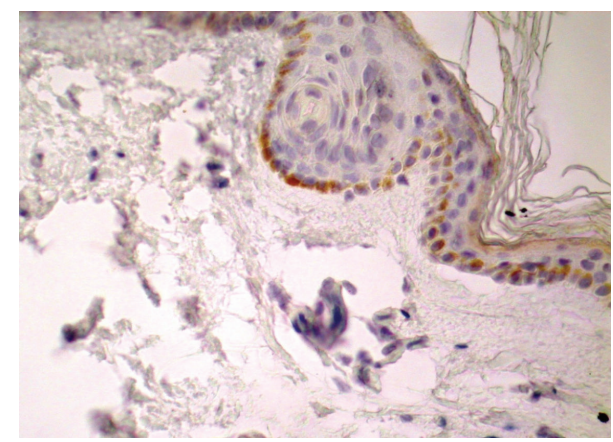

Figure 2: Expression of TF in epidermis. Perilesional skin. Bullous pemphigoid. 400x. Immunohistochemistry.

layers of the epidermis (in 19 out of 27 individuals), but expression insensitivity was lower.

Morphometric analysis showed a higher expression of TF in skin lesions than in perilesional skin in patients with BP $(p<0.02)$ (Table 1). Studies of patients with DH did not show a significant expression of TF either in pathologically changed tissues (TF was present in 3 out of 14 samples) or in perilesional skin (Figure 3). TF protein expression was also found in biopsy specimens from healthy individuals (in 2 out of 14). Therefore, the TF expression, in healthy group, was 


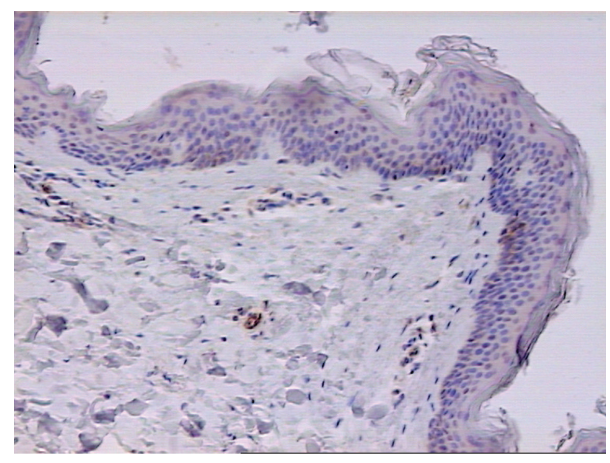

FIGURE 3: Lack of expression of TF. Healthy skin. 100x. Immunohistochemistry.

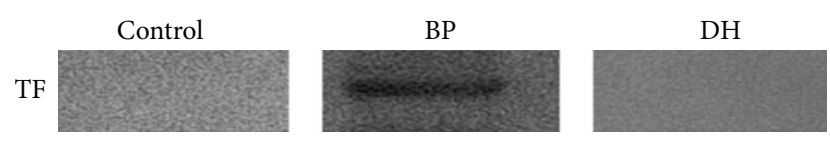

FIgURE 4: Tissue factor protein immunoblotting.

significantly lower than in the lesions in $\mathrm{BP}$ and comparable to expression in $\mathrm{DH}$ lesions.

3.2. Immunoblotting. Utilizing an immunoblotting technique and specific antibodies against TF we found significantly higher expression of TF in $\mathrm{BP}$ patients as compared to $\mathrm{DH}$ patients $(p<0.05)$ and as compared to healthy controls $(p<0.05)$ as shown in Figures 4 and 5.

\subsection{Immunoassay}

3.3.1. Tissue Factor. The mean TF plasma level in BP patients was $847.2 \pm 402.7 \mathrm{pg} / \mathrm{mL}$ as compared to patients with $\mathrm{DH}$ $(285.7 \pm 187.7 \mathrm{pg} / \mathrm{mL})$ and in the control groups, $252.6 \pm$ $169.3 \mathrm{pg} / \mathrm{mL}$ for BP and $221.9 \pm 153.3 \mathrm{pg} / \mathrm{mL}$ for $\mathrm{DH}$. These results indicate a statistically significant higher TF plasma concentration in patients with $\mathrm{BP}$ as compared to $\mathrm{DH}$ patients' and the control subjects $(p<0.001)$.

3.3.2. D Dimers. Prior to treatment, the $\mathrm{D}$ dimers levels in patients with $\mathrm{BP}$ were within the upper limits of the reference values (in 20 out of 27 patients). The mean $\mathrm{D}$ dimers level was $0.49 \pm 0.4 \mathrm{mg} / \mathrm{mL}$ (whereas the reference value range was $0.33-2.9 \mathrm{mg} / \mathrm{mL}$ ). In 7 patients these levels were elevated and were between 1.2 and $2.9 \mathrm{mg} / \mathrm{mL}$. These values were higher as compared to DH patients and the control group.

The mean $\mathrm{D}$ dimers values in $\mathrm{DH}$ patients were $0.31 \pm$ $0.19 \mathrm{mg} / \mathrm{mL}$ and were within the reference value. In the control group of patients, used as a reference group for BP patients, $\mathrm{D}$ dimers levels were within normal limits (the mean for control group was $0.29 \pm 0.3 \mathrm{mg} / \mathrm{mL}$ ). In the control group of patients used as a reference group for patients with $\mathrm{DH}$, $\mathrm{D}$ dimers levels were within normal limits; the mean was $0.34 \pm 0.6 \mathrm{mg} / \mathrm{mL}$. Therefore, there is a statistically significant difference in $\mathrm{D}$ dimers levels between the $\mathrm{DH}$ and BP patients $(p<0.05)$. There is also a statistically significant difference

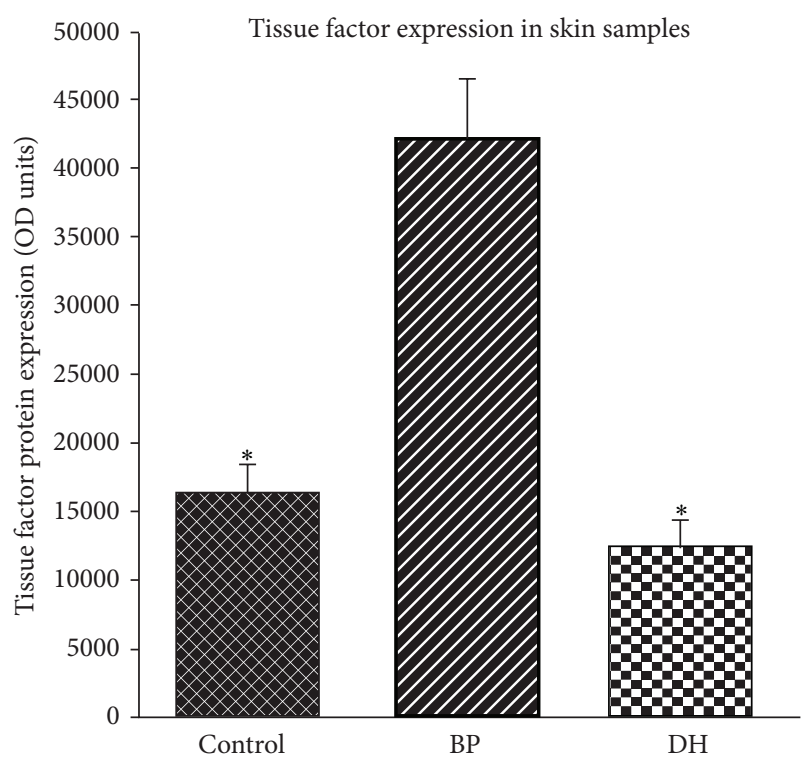

Figure 5: Mean value of optical densities (OD) of control and $\mathrm{DH}$ background and BP protein. Asterisks indicate a significance difference $\left({ }^{*} p<0.05\right)$ (mean \pm SEM), TF: tissue factor; $\mathrm{DH}$ : Duhring disease; BP: bullous pemphigoid.

in $\mathrm{D}$ dimers levels between $\mathrm{BP}$ patients and the control group $(p<0.05)$.

3.4. Coagulogram. In BP patients coagulation parameters were as follows: prothrombin time mean of $13.1 \pm 0.2 \mathrm{sec}$ (reference value: $12-18 \mathrm{sec}$ ), mean prothrombin index of $85.9 \% \pm 0.8 \%$ (reference range: $70-100 \%$ ), mean INR of $1.04 \pm$ 0.4 (reference range 0.9-1.30), and mean APTT of $31 \mathrm{~s} \pm$ $1.0 \mathrm{sec}$ (reference range: $26-36 \mathrm{sec}$.). In patients with $\mathrm{DH}$ coagulation parameters were as follows: mean prothrombin time of $15.3 \pm 0.5 \mathrm{sec}$, mean prothrombin index of $78.9 \% \pm$ $0.6 \%$, mean INR values of $1.28 \pm 0.3$, mean APTT of $29 \mathrm{sec} \pm$ $1.0 \mathrm{sec}$ (reference range: $26-36 \mathrm{sec}$ ), and average values of $29 \mathrm{~s}$ $\pm 1.0 \mathrm{sec}$.

In the control group used for comparison with $\mathrm{BP}$ patients, coagulation parameters were as follows: mean prothrombin time of $14.2 \pm 0.2 \mathrm{sec}$, mean prothrombin index of $88.7 \% \pm 0.4$, mean INR of $1.17 \pm 0.5$, and mean APTT of $30 \mathrm{sec} \pm 1.0 \mathrm{sec}$. In the control group used for comparison with DH patients, coagulation parameters were as follows: mean prothrombin time of $14.9 \pm 0.5 \mathrm{sec}$, mean prothrombin index of $98.7 \% \pm 0.2$, mean INR of $1.21 \pm 0.4$, and mean APTT of $28 \mathrm{sec} . \pm 1.0 \mathrm{sec}$.

There is no difference in coagulation parameters between $\mathrm{BP}$ and DH groups and examined groups and healthy individuals. The platelets levels in all groups were within the reference range (BP patients: $284.0 \pm 96.9 \times 10^{9} / \mathrm{L} ; \mathrm{DH}$ patients: $187.0 \pm 58.4 \times 10^{9} / \mathrm{L}$; and control group: $254.0 \pm 79 \times$ $\left.10^{9} / \mathrm{L}\right)$.

3.4.1. Fibrinogen Levels. In $\mathrm{BP}$ patients, mean fibrinogen level was $4.50 \pm 0.18 \mathrm{~g} / \mathrm{L}$ as compared to $2.60 \pm 0.3 \mathrm{~g} / \mathrm{L}$ in the 
control group. DH patients mean fibrinogen concentration was $2.80 \pm 0.14 \mathrm{~g} / \mathrm{L}$ as compared to $2.45 \pm 0.26 \mathrm{~g} / \mathrm{L}$ in the control group. There is a statistically significant difference $(p<0.05)$ between the group of patients with DH and BP, as well as between BP patients and the control group $(p<0.05)$.

3.4.2. DD, F2, F5, F7, and Venom Measurements by Destiny Plus System in BP Patients. The D dimers levels were significantly higher in the BP patient group as compared to controls $(312 \mathrm{ng} / \mathrm{mL}$ versus $96 \mathrm{ng} / \mathrm{mL})(p<0.01)$. All blood coagulation factors like F2, F5, F7, and venom in BP and DH patients and control group were within the reference values. The following correlations were found in BP patients between the following: F2 and DD $(r=-0.3419, p=0.003)$; F5 and $\mathrm{DD}(r=-0.2074, p=0.005)$; DD and venom $(r=+0.4120$, $p=0.0111)$; F5 and F2 $(r=+0.4258, p=0.0031)$; F7 and F2 $(r=+0.6937, p=0.00001)$. Similarly, they were found between the following: venom and F2 $(r=-0.5517$, $p=0.0001)$; venom and F5 $(r=-0.3985, p=0.0054)$; venom and F7 $(r=-0.4424, p=0.0016)$ (Figure 6).

\section{Discussion}

Eosinophils in response to many stimuli secrete many proteins including eosinophil cationic protein (ECP). Elevated ECP prolongs the clotting time, due to its antagonism with heparin and streptokinase [23]. Another protein, playing an important role in coagulation process, is tissue factor (TF), also produced by eosinophils. Glucocorticoids do not stop the release of these proteins [24], but immunosuppressive agents such as cyclosporine inhibit the secretion of ECP from eosinophils.

Marzano et al. [13] in their study evaluated the TF expression in skin biopsies in BP patients and showed that immunohistochemistry revealed strong TF reactivity in BP skin, and colocalization studies confirmed eosinophils as a source of TF. Our results indicate a statistically significant difference in the value of TF between the group of patients with $\mathrm{DH}$ and $\mathrm{BP}$ and $\mathrm{BP}$ and the control group.

Elevated levels of the prothrombin fragment $\mathrm{F} 1+2$ and both plasma and blister fluid of BP patients in the active phase of the disease were reported [13]. During the remission, the concentration of coagulation activation markers was normal. The concentration of the prothrombin fragment $\mathrm{F} 1+2$ correlated with the concentration of immunoglobulins directed against the BP180 antigen [13].

$\mathrm{TF}$ is the main activator of extrinsic coagulation pathway, which in addition to well-documented prothrombotic properties plays an important role in the inflammatory process [17]. It is a recognized factor connecting the immune system with coagulation system. Expression of TF present in fibroblasts, epithelial and endothelial cells, and macrophages is induced by antigen-antibody complexes, complement activation products, proteins, and proinflammatory cytokines [22]. Our study showed low level TF expression in DH patients and healthy subjects, confirming its constitutive production.

Cugno et al. [22] emphasized the role of TNF and IL-6, which are increased in $\mathrm{BP}$, in the induction of TF expression.
The eosinophilic infiltration in BP skin might provoke an elevation of TF concentration in this disease. Morphometric analysis showed a higher expression of TF in skin lesion than in perilesional skin in patients with $\mathrm{BP}$ opposite to $\mathrm{DH}$ and control biopsies. In our study, we confirmed that kind of influx has important role in activation of TF in skin.

The inflammatory and neoplastic processes of coagulation and fibrinolysis system are activated in response to the local infiltration of inflammatory cells and increased vascular permeability $[18,22]$. TF increases the influx of monocytes to the sites of inflammation $[25,26]$, and its induction in monocytes may depend on a direct interaction with $\mathrm{T}$ cells. Several studies have confirmed the effect of the inflammatory process in the excessive production of tissue factor [27-30].

Both in the case of pemphigoid and $\mathrm{DH}$ in our earlier studies, we showed in skin lesions increased expression of proinflammatory cytokines which may activate the production of TF. However, only in BP, expression was increased in both: skin lesions and blood. This is probably related to eosinophilic infiltration in BP and production by these cells procoagulant factors [17].

The product of activation of TF, thrombin, acts by increasing vascular permeability for inflammatory cells and causing the release of interleukin production and activation of adhesion molecules [22]. In our earlier studies, we confirmed the increased expression of adhesins and MMPs, which may be an effect of action of TF in BP [31].

In the aspect of described results, participation of activation markers of eosinophils, mast cells activation, and coagulation factors in the pathogenesis of some subepidermal blistering diseases appears to be particularly interesting [25, 26].

Abnormal expression of procoagulant factors in the tissue may be responsible for the inflammatory process and blister formation in BP. According to literature [32, 33], up to $25 \%$ of the cells in the DH influx are eosinophils. In addition, myeloperoxidase, ECP, and major basic protein secreted by eosinophils were found in sera of patients with $\mathrm{DH}$, which may indicate the production of inflammatory mediators by eosinophils in $\mathrm{DH}$ [33]. The small TF expression in our study in $\mathrm{DH}$ patients may be related to the fact that the presence of a small population of eosinophils in the inflammatory infiltrates in these patients.

This study showed that the group of BP patients revealed the normal mean $\mathrm{D}$ dimer concentration, however the individual results were within the upper limit of the reference range, and some patients had elevated D dimer levels. However, in the evaluation by the Destiny Plus System, $\mathrm{D}$ dimer concentration was significantly higher in the BP patient group as compared to controls. It should be noted that these values were determined prior to treatment with corticosteroids, which exacerbate prothrombotic processes. The values of $\mathrm{D}$ dimer in patients with $\mathrm{DH}$ were normal and similar to the levels in healthy subjects.

The treatment of patients with BP, especially the elderly, with local steroids or antihistamines and immunomodulators has been postulated $[34,35]$. Therefore, it seems important to indicate the need to change the current treatment of patients with BP with the standard treatment of corticosteroid 


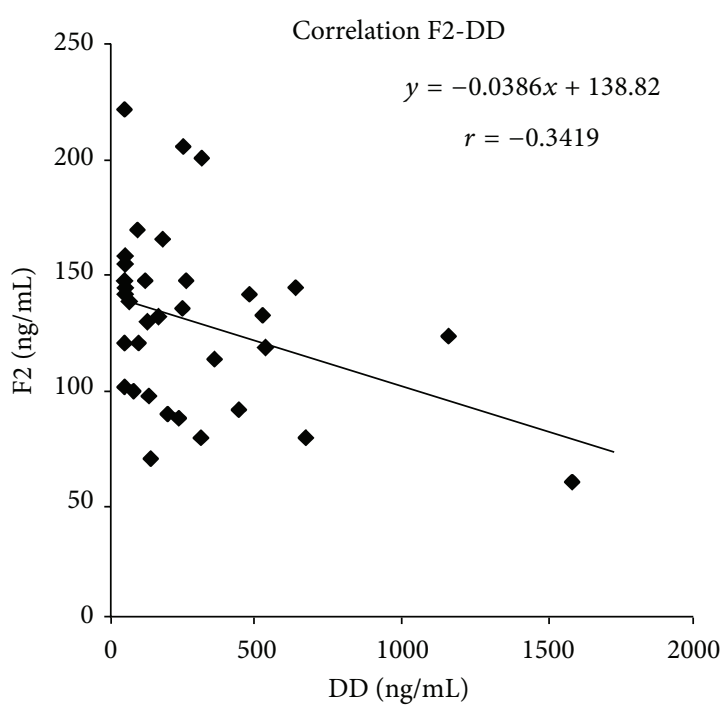

(a)

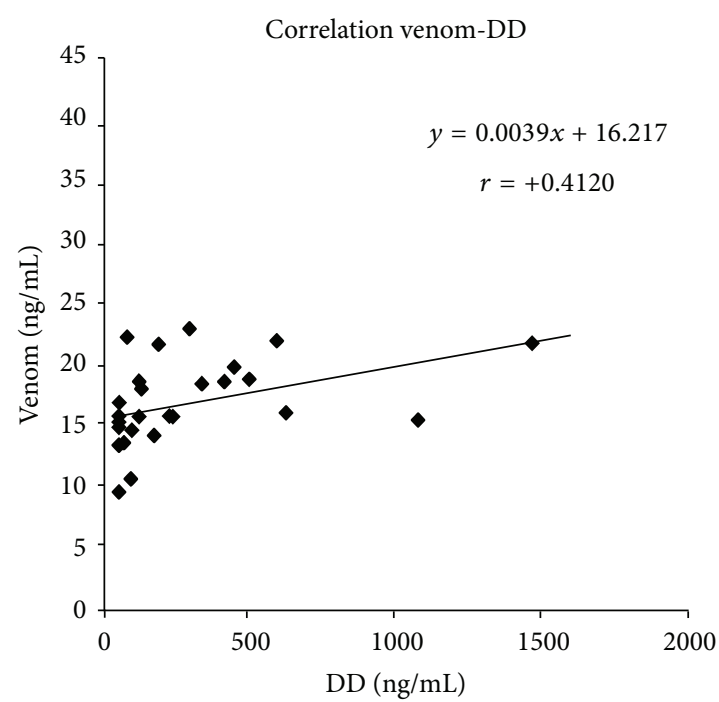

(c)

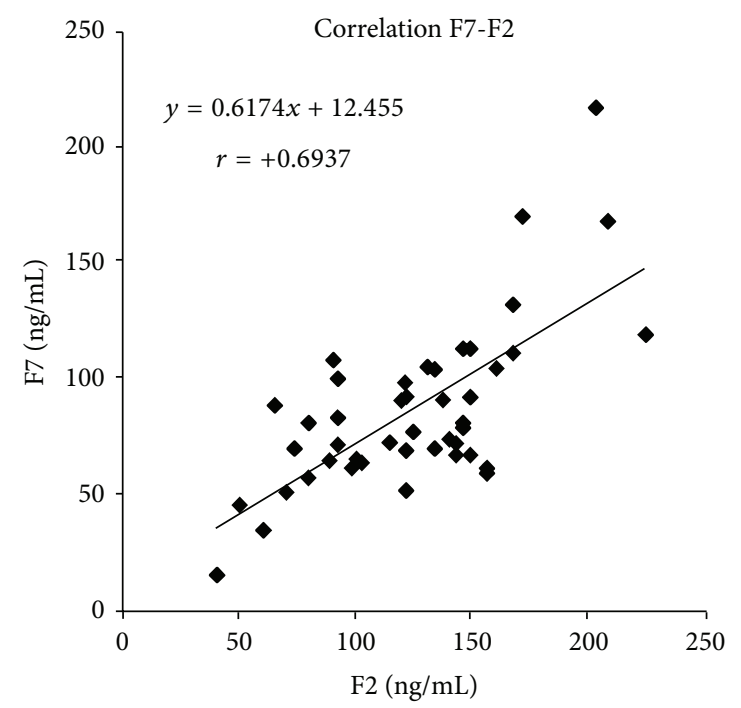

(e)

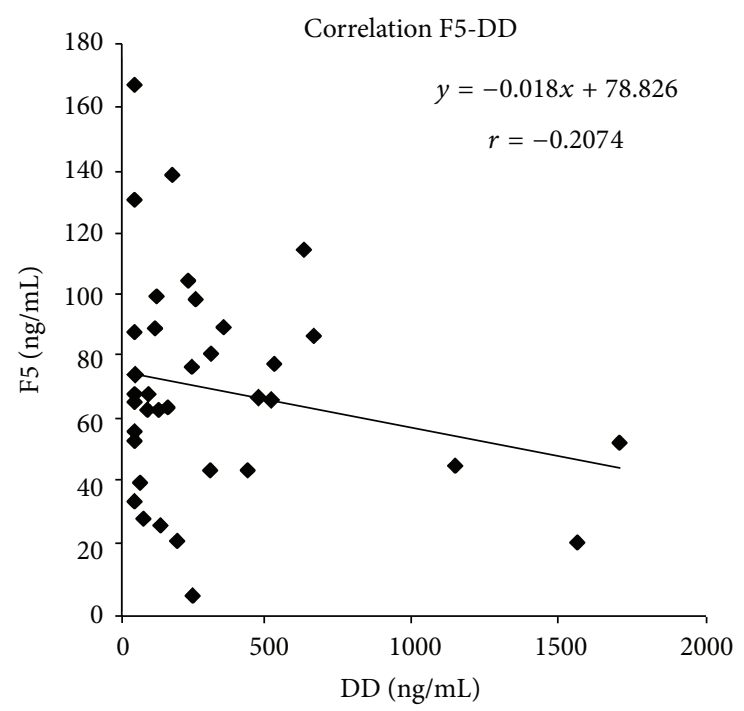

(b)

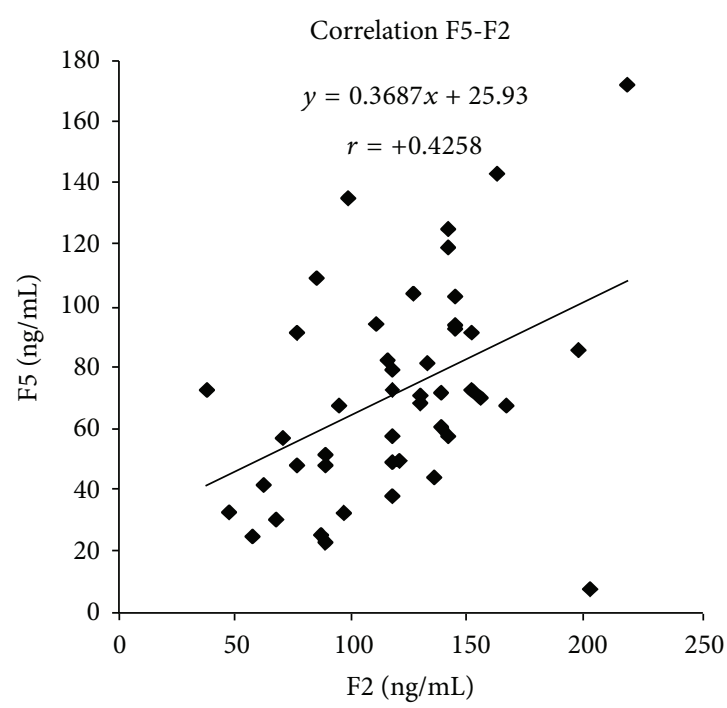

(d)

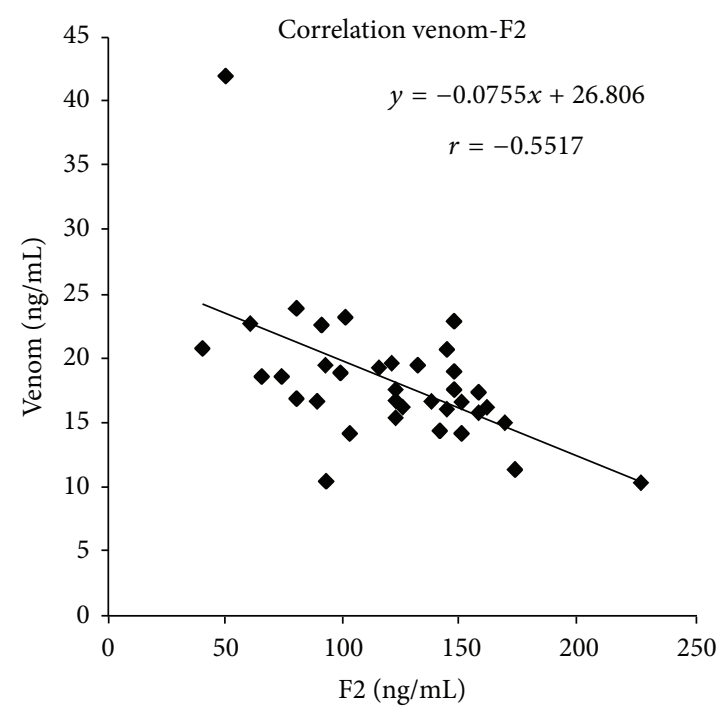

(f)

Figure 6: Continued. 


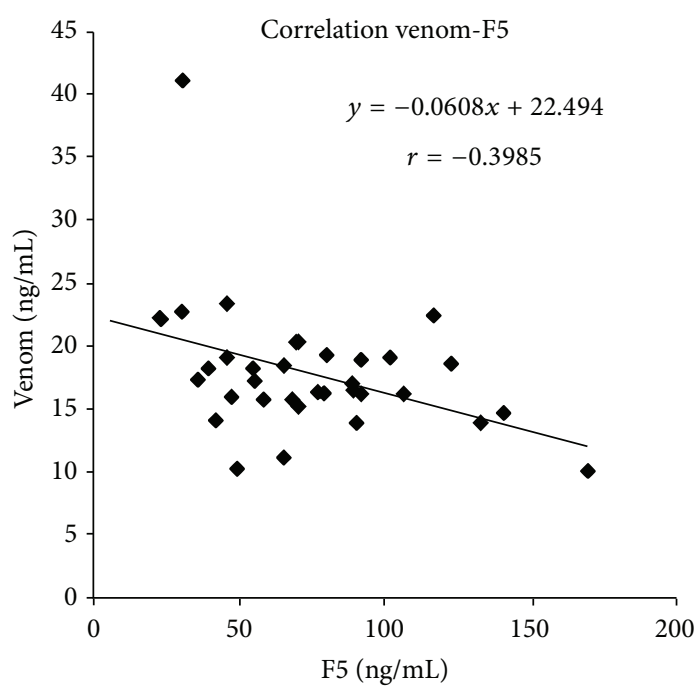

(g)

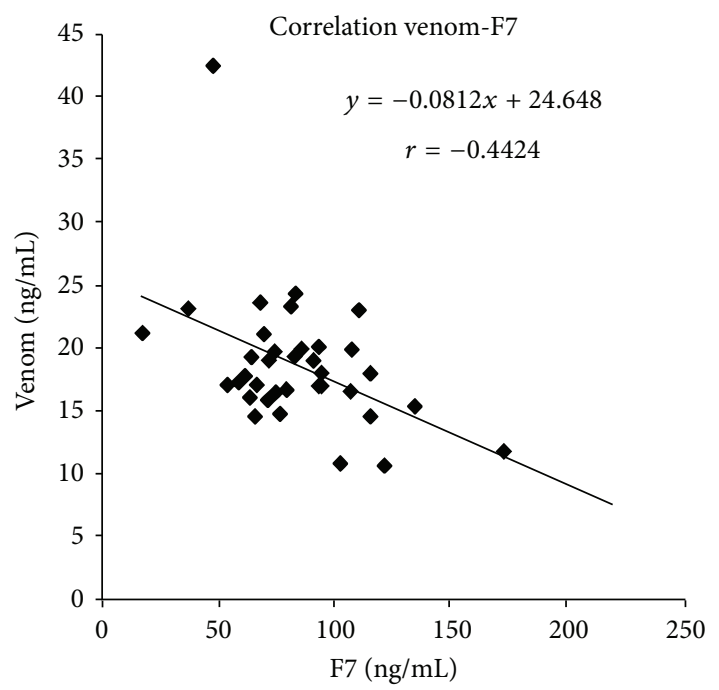

(h)

FIGURE 6: F2, F5, F7, D dimers, and venom: correlations in BP patients.

into drugs that do not increase the risk of thrombotic complications. It is important in planning treatment preventing complications.

In the therapy of pemphigoid, we use many drugs acting through different mechanisms. Because of the key role of autoimmune phenomena, most commonly used drugs are glucocorticosteroids (GCS), a class of drugs having a particularly strong impact on the homeostatic system [36]. Effects of thrombogenicity related to GCS might be connected with their direct influence on the coagulation system and the metabolic disturbances linked to the nature of the disease [37]. Our study also showed elevated levels of fibrinogen in patients with BP compared to the patient with $\mathrm{DH}$ and control group. Some clinical studies have shown that fibrinogen level above $3.5 \mathrm{~g} / \mathrm{L}$ is seen as the value that causes an increased risk of thrombosis and thus an increased risk of ischemic heart disease or a stroke $[38,39]$. The main cause of death in patients with pemphigoid is the complications of steroid treatment, which is considered to be the most effective in this disease [40-42]. Rzany et al. [43] underline that embolic complications with severe bacterial infections are the most common side effects in the course of corticosteroid overall in the age group above 70 years. Other authors also confirm the good results of local therapy in the treatment of bullous pemphigoid and reducing the incidence of adverse events compared with patients treated with oral steroids [44].

So far, there are no data on the expression of procoagulant factors and abnormal activation of coagulation process in the formation of blisters. However, the dominant role of eosinophils in the inflammatory infiltration in BP was recently revealed and the fact that they secrete tissue factor gives the scientific basis to consider the contribution of this process in the pathogenesis of lesions in the subepidermal bullous diseases. Demonstration of elevated D dimer and prothrombin in patients in the active phase of the disease indicates the need to assess the suitability of patients for thromboembolic complications. An increased risk of death due to the stroke in patients with BP treated with glucocorticoids might confirm these findings. Despite these risks, the latest consensus on the treatment of pemphigus and of bullous pemphigoid still recommends the use of corticosteroids in the treatment of BP [45].

Our results may be useful in clinical practice and objective determination of the degree of disease activity as well as an important therapeutic indication which allows reducing the high mortality rate among patients with pemphigoid treated with corticosteroid and confirming a trend in the treatment of this disease with antihistamines and nonsteroidal immunomodulators.

\section{Conflict of Interests}

The authors have no conflict of interests to declare.

\section{Acknowledgments}

The study was funded by the research projects of Medical University of Lodz no. 503/1-152-01/503-01 and The Polish Science Committee Grant no. 4746/B/PO1/2009/37.

\section{References}

[1] S. Kárpáti, "Dermatitis herpetiformis: close to unravelling a disease," Journal of Dermatological Science, vol. 34, no. 2, pp. 8390, 2004.

[2] M. Sárdy, S. Kárpáti, B. Merkl, M. Paulsson, and N. Smyth, "Epidermal transglutaminase (TGase 3) is the autoantigen of dermatitis herpetiformis," The Journal of Experimental Medicine, vol. 195, no. 6, pp. 747-757, 2002.

[3] W. Dieterich, E. Laag, L. Bruckner-Tuderman et al., "Antibodies to tissue transglutaminase as serologic markers in patients with 
dermatitis herpetiformis," Journal of Investigative Dermatology, vol. 113, no. 1, pp. 133-136, 1999.

[4] J. D. Hendrix, K. L. Mangum, J. J. Zone, and W. R. Gammon, "Cutaneous IgA deposits in bullous diseases function as ligands to mediate adherence of activated neutrophils," Journal of Investigative Dermatology, vol. 94, no. 5, pp. 667-672, 1990.

[5] M. V. Dahl, R. J. Falk, R. Carpenter, and A. F. Michael, "Membrane attack complex of complement in dermatitis herpetiformis," Archives of Dermatology, vol. 121, no. 1, pp. 70-72, 1985.

[6] K. Airola, M. Vaalamo, T. Reunala, and U. K. Saarialho-Kere, "Enhanced expression of interstitial collagenase, stromelysin1 , and urokinase plasminogen activator in lesions of dermatitis herpetiformis," Journal of Investigative Dermatology, vol. 105, no. 2, pp. 184-189, 1995.

[7] R. F. Ghohestani, J. Novotney, M. Chaudhary, and R. S. Agah, "Bullous pemphigoid: from the bedside to the research laboratory," Clinics in Dermatology, vol. 19, no. 6, pp. 690-696, 2001.

[8] J. R. Stanley, "Cell adhesion molecules as targets of autoantibodies in pemphigus and pemphigoid, bullous diseases due to defective epidermal cell adhesion," Advances in Immunology, vol. 53, pp. 291-325, 1993.

[9] R. E. Jordon, E. H. Beutner, E. Witebsky, G. Blumental, W. L. Hale, and W. F. Lever, "Basement zone antibodies in bullous pemphigoid," Journal of the American Medical Association, vol. 200, no. 9, pp. 751-756, 1967.

[10] E. Schmidt, S. Reimer, N. Kruse et al., "Autoantibodies to BP180 associated with bullous pemphigoid release interleukin- 6 and interleukin-8 from cultured human keratinocytes," Journal of Investigative Dermatology, vol. 115, no. 5, pp. 842-848, 2000.

[11] A. Zebrowska, M. Wągrowska-Danilewicz, M. Danilewicz et al., "Expression of selected ADAMs in bullous pemphigoid and dermatitis herpetiformis," Journal of Dermatological Science, vol. 56, no. 1, pp. 58-61, 2009.

[12] M. Cugno, A. V. Marzano, P. Bucciarelli et al., "Increased risk of venous thromboembolism in patients with bullous pemphigoid," Thrombosis and Haemostasis, vol. 114, no. 6, 2015.

[13] A. V. Marzano, A. Tedeschi, D. Fanoni et al., "Activation of blood coagulation in bullous pemphigoid: role of eosinophils, and local and systemic implications," British Journal of Dermatology, vol. 160, no. 2, pp. 266-272, 2009.

[14] M. J. Rico, C. Benning, E. S. Weingart, R. D. Streilein, and R. P. Hall III, "Characterization of skin cytokines in bullous pemphigoid and pemphigus vulgaris," British Journal of Dermatology, vol. 140, no. 6, pp. 1079-1086, 1999.

[15] L. Engineer, K. Bhol, S. Kumari, and A. Razzaque Ahmed, "Bullous pemphigoid: interaction of interleukin 5, anti-basement membrane zone antibodies and eosinophils. A preliminary observation," Cytokine, vol. 13, no. 1, pp. 32-38, 2001.

[16] B. O. Gerber, M. P. Zanni, M. Uguccioni et al., "Functional expression of the eotaxin receptor CCR3 in T lymphocytes colocalizing with eosinophils," Current Biology, vol. 7, no. 11, pp. 836-843, 1997.

[17] C. Moosbauer, E. Morgenstern, S. L. Cuvelier et al., "Eosinophils are a major intravascular location for tissue factor storage and exposure," Blood, vol. 109, no. 3, pp. 995-1002, 2007.

[18] Y. A. Muller, M. H. Ultsch, R. F. Kelley et al., "Structure of the extracellular domain of human tissue factor: location of the factor VIIa binding sile," Biochemistry, vol. 33, pp. 10864-10867, 1994.
[19] A. V. Marzano, A. Tedeshi, D. Spinelli, D. Fanoni, C. Crosti, and M. Cugno, "Coagulation activation in autoimmune bullous diseases," Clinical \& Experimental Immunology, vol. 158, no. 1, pp. 31-36, 2009.

[20] A. V. Marzano, A. Tedeschi, I. Polloni, C. Crosti, and M. Cugno, "Prothrombotic state and impaired fibrinolysis in bullous pemphigoid, the most frequent autoimmune blistering disease," Clinical and Experimental Immunology, vol. 171, no. 1, pp. 7681, 2013.

[21] M. A. Cunningham, P. Romas, P. Hutchinson, S. R. Holdsworth, and P. G. Tipping, "Tissue factor and factor VIIa receptor/ligand interactions induce proinflammatory effects in macrophages," Blood, vol. 94, no. 10, pp. 3413-3420, 1999.

[22] M. Cugno, A. Tedeschi, R. Asero, P. L. Meroni, and A. V. Marzano, "Skin autoimmunity and blood coagulation," Autoimmunity, vol. 43, no. 2, pp. 189-194, 2010.

[23] P. Venge, J. Byström, M. Carlson et al., "Eosinophil cationic protein (ECP): molecular and biological properties and the use of ECP as a marker of eosinophil activation in disease," Clinical and Experimental Allergy, vol. 29, no. 9, pp. 1172-1186, 1999.

[24] H. Kita, R. Abu-Ghazaleh, C. J. Sanderson, and G. J. Gleich, "Effect of steroids on immunoglobulin-induced eosinophil degranulation," The Journal of Allergy and Clinical Immunology, vol. 87, no. 1, pp. 70-77, 1991.

[25] G. Del Prete, M. De Carli, R. M. Lammel et al., "Th1 and Th2 Thelper cells Ebert opposite regulatory effects on procoagulant activity and tissue factor production by human monocytes," Blood, vol. 86, no. 1, pp. 250-257, 1995.

[26] I. Kucharewicz, K. Kowal, W. Buczko, and A. BodzentaŁukaszyk, "The plasmin system in airway remodeling," Thrombosis Research, vol. 112, no. 1-2, pp. 1-7, 2003.

[27] A. K. So, P. A. Varisco, B. Kemkes-Matthes et al., "Arthritis is linked to local and systemic activation of coagulation and fibrinolysis pathways," Journal of Thrombosis and Haemostasis, vol. 1, no. 12, pp. 2510-2515, 2003.

[28] K. Kume, M. Yamasaki, M. Tashiro, I. Yoshikawa, and M. Otsuki, "Activations of coagulation and fibrinolysis secondary to bowel inflammation in patients with ulcerative colitis," Internal Medicine, vol. 46, no. 17, pp. 1323-1330, 2007.

[29] M. Levi, T. van der Poll, H. Ten Cate, and S. J. H. van Deventer, "The cytokine-mediated imbalance between coagulant and anticoagulant mechanisms in sepsis and endotoxaemia," European Journal of Clinical Investigation, vol. 27, no. 1, pp. 3-9, 1997.

[30] P. C. Y. Liaw, C. T. Esmon, K. Kahnamoui et al., "Patients with severe sepsis vary markedly in their ability to generate activated protein C," Blood, vol. 104, no. 13, pp. 3958-3964, 2004.

[31] J. Narbutt, E. Waszczykowska, J. Lukamowicz, A. SysaJedrzejowska, J. Kobos, and A. Zebrowska, "Disturbances of the expression of metalloproteinases and their tissue inhibitors cause destruction of the basement membrane in pemphigoid," Polish Journal of Pathology, vol. 57, no. 2, pp. 71-76, 2006.

[32] M. Caproni, C. Cardinalli, A. D’Agata, W. Selvaggi, and P. Fabbri, "Serum eosinophil cationic protein, myeloperoxidase, tryptase, eotaxin and Th2-L-like cytokines in dermatitis herpetiformis," International Archives of Allergy and Immunology, vol. 128, no. 1, pp. 67-72, 2002.

[33] E. Marietta, K. Black, M. Camilleri et al., "A new model for dermatitis herpetiformis that uses HLA-DQ8 transgenic NOD mice," Journal of Clinical Investigation, vol. 114, no. 8, pp. 10901097, 2004.

[34] S. M. Langan, R. Hubbard, K. Fleming, and J. West, "A population-based study of acute medical conditions associated 
with bullous pemphigoid," British Journal of Dermatology, vol. 161, no. 5, pp. 1149-1152, 2009.

[35] S. M. Langan, L. Smeeth, R. Hubbard, K. M. Fleming, C. J. P. Smith, and J. West, "Bullous pemphigoid and pemphigus vulgaris-incidence and mortality in the UK: population based cohort study," The British Medical Journal, vol. 337, no. 7662, pp. 160-163, 2008.

[36] D. J. Brotman, J. P. Girod, A. Posch et al., "Effects of short-term glucocorticoids on hemostatic factors in healthy volunteers," Thrombosis Research, vol. 118, no. 2, pp. 247-252, 2006.

[37] B. Van Zaane, E. Nur, A. Squizzato et al., "Systematic review on the effect of glucocorticoid use on procoagulant, anti-coagulant and fibrinolytic factors," Journal of Thrombosis and Haemostasis, vol. 8, no. 11, pp. 2483-2493, 2010.

[38] E. Ernst, "Plasma fibrinogen - an independent cardiovascular risk factor," Journal of Internal Medicine, vol. 227, no. 6, pp. 365372, 1990.

[39] R. M. Senior, W. F. Skogen, G. L. Griffin, and G. D. Wilner, "Effects of fibrinogen derivatives upon the inflammatory response. Studies with human fibrinopeptide B," Journal of Clinical Investigation, vol. 77, no. 3, pp. 1014-1019, 1986.

[40] E. Schmidt, E.-B. Bröcker, and M. Goebeler, "Rituximab in treatment-resistant autoimmune blistering skin disorders," Clinical Reviews in Allergy and Immunology, vol. 34, no. 1, pp. 56-64, 2008.

[41] J. A. Fairley, C. L. Baum, D. S. Brandt, and K. A. N. Messingham, "Pathogenicity of IgE in autoimmunity: successful treatment of bullous pemphigoid with omalizumab," The Journal of Allergy and Clinical Immunology, vol. 123, no. 3, pp. 704-705, 2009.

[42] P. Joly, J. Benichou, C. Lok et al., "Prediction of survival for patients with bullous pemphigoid: a prospective study," Archives of Dermatology, vol. 141, no. 6, pp. 691-698, 2005.

[43] B. Rzany, K. Partscht, M. Jung et al., "Risk factors for lethal outcome in patients with bullous pemphigoid: low serum albumin level, high dosage of glucocorticosteroids, and old age," Archives of Dermatology, vol. 138, no. 7, pp. 903-908, 2002.

[44] A. Stockman, H. Beele, Y. Vanderhaeghen, and J. M. Naeyaert, "Topical class I corticosteroids in 10 patients with bullous pemphigoid: correlation of the autcome with the severity degree of the disease and review of the literature," Journal of the European Academy of Dermatology and Venereology, vol. 18, no. 2, pp. 164-168, 2004.

[45] H. Y. Schultz, L. A. Diaz, D. A. Sirois, V. P. Werth, and S. A. Grando, "Generating consensus research goals and treatment strategies for pemphigus and pemphigoid: the 2010 JC Bystryn pemphigus and pemphigoid meeting," Journal of Investigative Dermatology, vol. 131, no. 7, pp. 1395-1399, 2011. 


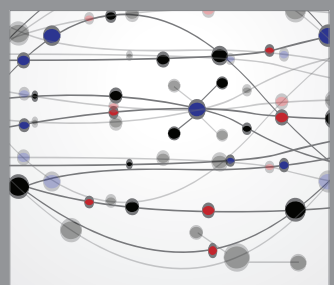

The Scientific World Journal
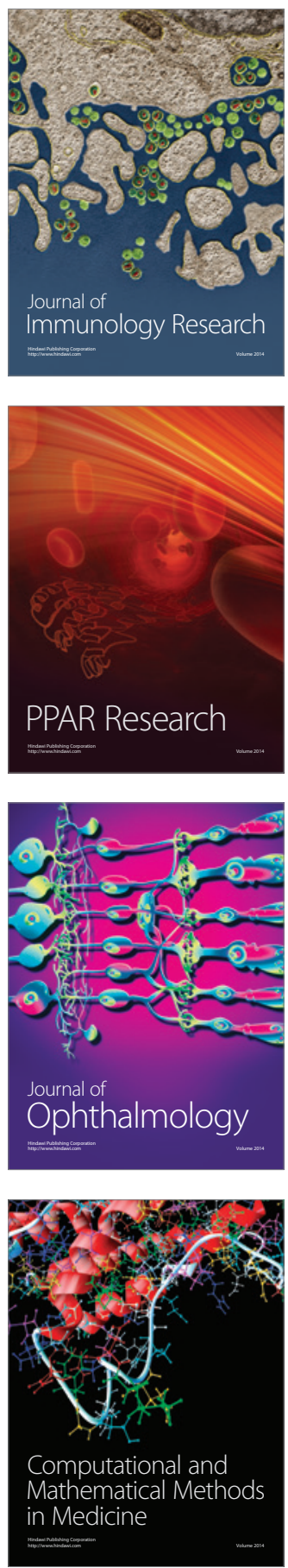

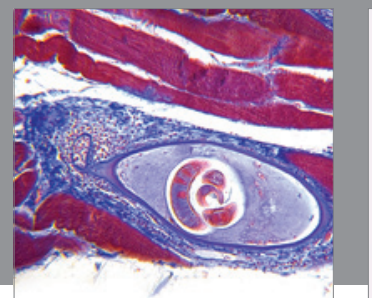

Gastroenterology

Research and Practice
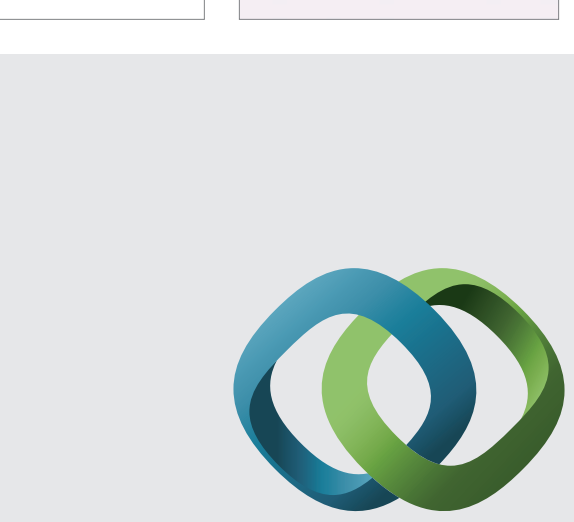

\section{Hindawi}

Submit your manuscripts at

http://www.hindawi.com
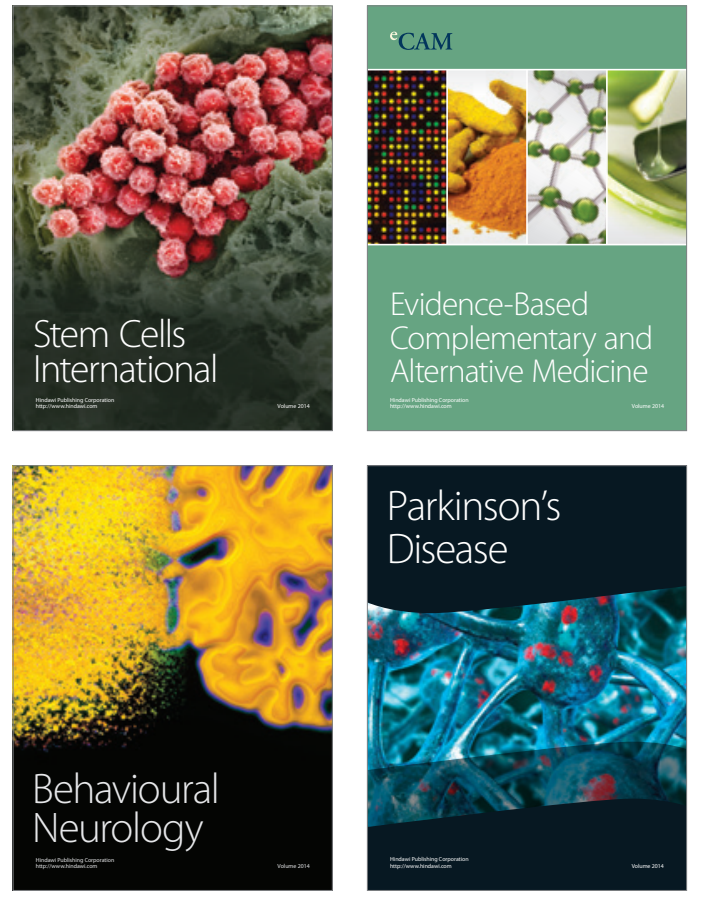
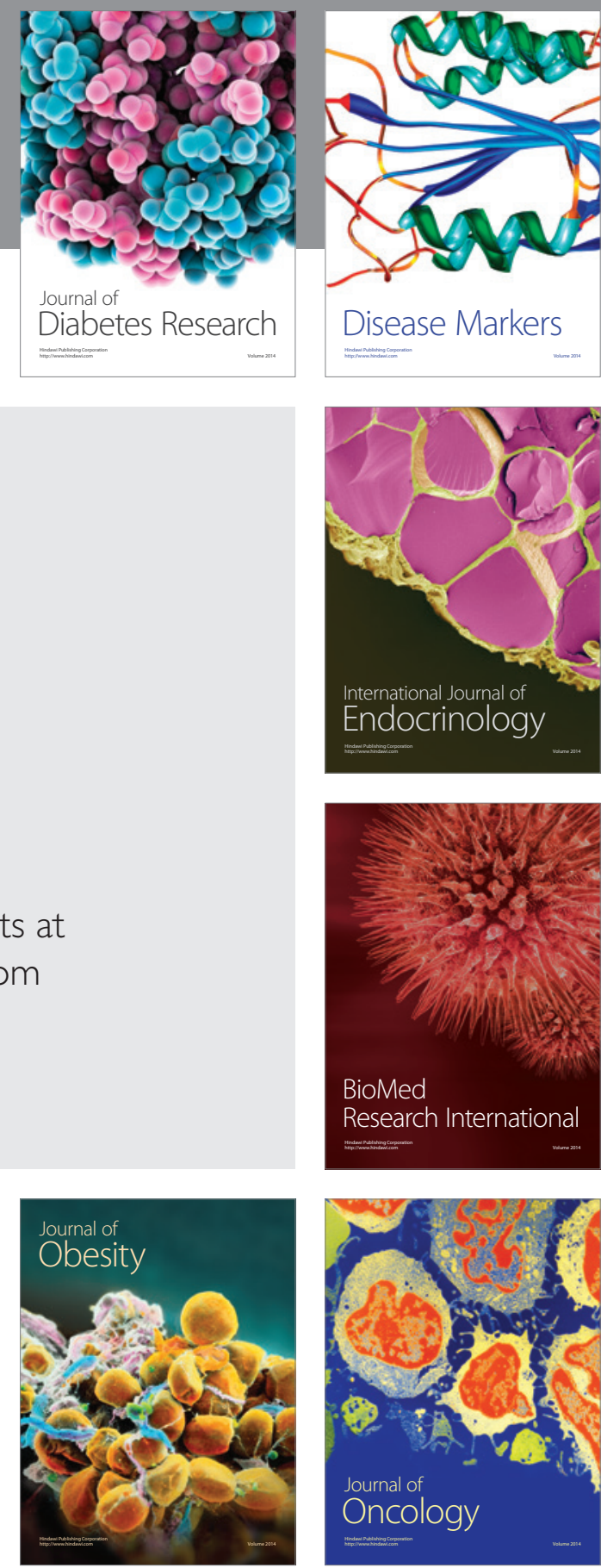

Disease Markers
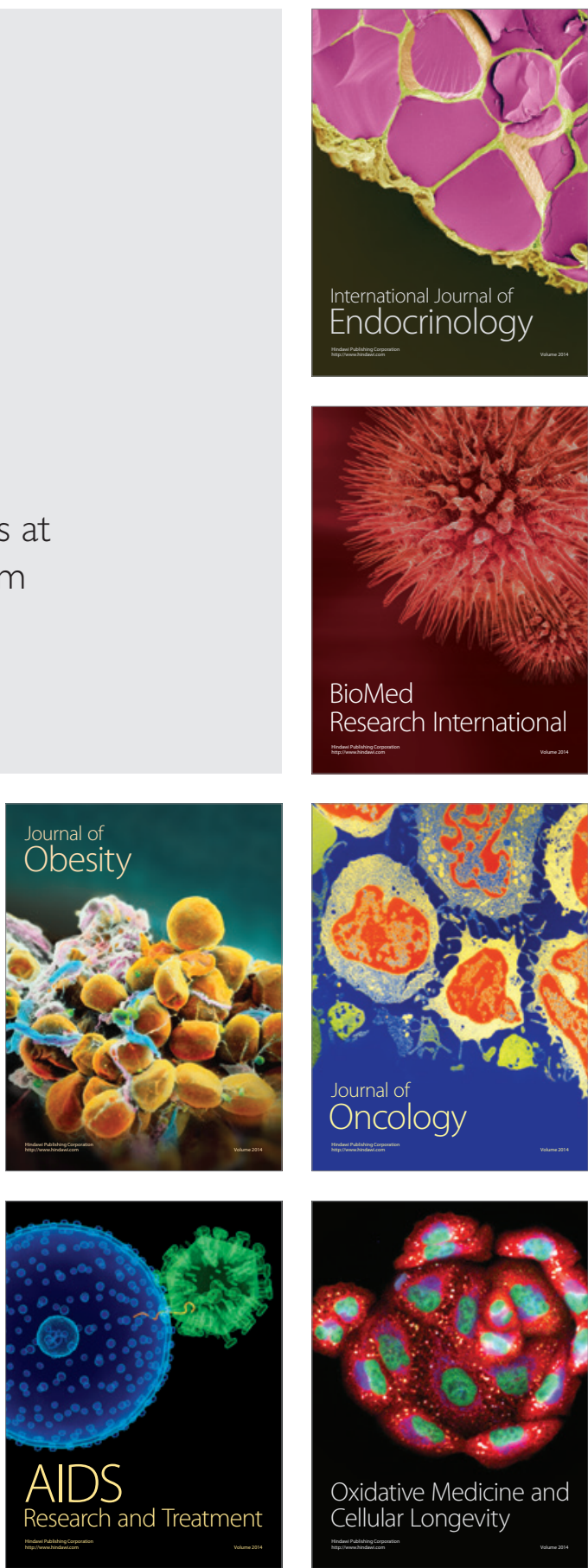